\title{
ORIGINAL
}

\section{VARIABLES QUE CONDICIONAN LA UTILIZACIÓN DE LA CONSULTA DE ENFERMERÍA EN CENTROS DE SALUD DE LA COMUNIDAD DE MADRID (*)}

\author{
Jesús Martín-Fernández (1), Gemma Rodríguez-Martínez (2), Gloria Ariza-Cardiel (3), Mª Angeles Ver- \\ gel Gutierrez (4), Ana Victoria Hidalgo Escudero (5) y Juan Francisco Conde-López (6).
}

(1) Consultorio de Villamanta (CS Navalcarnero). Gerencia de Atención Primaria. Madrid.

(2) Centro de Salud Condes de Barcelona. Gerencia de Atención Primaria. Madrid.

(3) Unidad Docente Multiprofesional de Atención Familiar y Comunitaria Oeste. Unidad de Apoyo a la Investigación. Gerencia de Atención Primaria. Madrid.

(4) Centro de Salud Navalcarnero. Gerencia de Atención Primaria. Madrid.

(5) Centro de Salud Ramón y Cajal. Gerencia de Atención Primaria. Madrid.

(6) Centro de Salud Laín Entralgo. Gerencia de Atención Primaria. Madrid.

(*) La realización del trabajo de campo de este estudio fue posible gracias a las ayudas para la realización de proyectos de investigación en el campo de resultados en salud en Atención Primaria establecida en la Orden 472/2010, de 16 de septiembre, de la Consejería de Sanidad. Comunidad de Madrid.

\section{RESUMEN}

Fundamentos: Diferentes condiciones de utilización de servicios sanitarios pueden originar situaciones de falta de equidad. El objetivo del trabajo es conocer si existen diferencias en el uso de la consulta de enfermería en atención primaria.

Métodos: Estudio multicéntrico transversal en 23 centros de salud de la Comunidad de Madrid. Se incluyó a 662 sujetos. Se recogieron variables relativas al centro de salud, de la consulta de enfermería, características sociodemograficas y de necesidad en salud. Se estudiaron la calidad de vida y la satisfacción de los sujetos. Las variables se clasificaron, según el modelo "conductual", en predisponentes, facilitadoras o de necesidad. Se construyeron modelos multivariantes explicativos.

Resultados: Vivir en zonas de renta alta y la edad se asociaron con aumentos del $17 \%$ (IC95\%: 0,4-36,9\%) y del $11,0 \%$ por cada década (IC95\%: $6,2-16,2)$ de las consultas/año de enfermería. De los factores facilitadores, cada minuto añadido de duración de la consulta se asoció con un aumento del $2,0 \%$ (IC95\%:1,2-2,9\%) en las consultas/año, cada nueva consulta médica se asociaba con un aumento del 2,7\% (IC95\%: 2,1-3,2\%) y la demora en conseguir cita superior a un día supuso una disminución del 32,8\% (IC95\%: 19,3$44,1 \%$ ) del número de consultas. Cada condición crónica, que expresa necesidad en salud, se asoció con un incremento del número de consultas del 4,8\% (IC 95\%: 1,7-8,0\%). La mejor percepción de la calidad de vida se asoció con una reducción de las consultas de un 5,4\% (IC95\%: 1,0-8,7\%)

Conclusión: Las diferencias en el uso de la consulta de enfermería responde a criterios de necesidad, pero también está influenciada por condiciones de accesibilidad.

Palabras clave: Servicios sanitarios Necesidades y demanda. Utilización. Atención primaria de la salud. Enfermería. Inequidad. Factores socioeconómicos.

\section{Correspondencia}

Jesús Martín-Fernández

Centro de Salud Villamanta

Avda de la Libertad, 7.

28610 Villamanta

Madrid

jmfernandez@salud.madrid.org

\section{ABSTRACT \\ Differences in the Nursing Consultation Utilization in Primary Care, Spain}

Background: Different conditions in health services utilization may create situations of inequity. The objective was analyze the differences of nurse consultation utilization in primary care.

Methods: Cross-sectional study, in 23 health centres in Madrid. Environmental variables, consultation characteristics, socio-demographic and health need characteristics were collected. The quality of life and satisfaction were also studied. The variables were classified according to the "behavioral model" in predisposing, enabling or need variables. Explanatory multivariate models were constructed (Generalized-EstimatingEquations).

Results: The higher income areas and aging, predisposing factors, were associated with increases of $17 \%(95 \%$ CI: 0.4 to $36.9 \%)$ and $11.0 \%$ per decade ( $95 \%$ CI: 6.2 - 16.2) in nursing consultations per year. Among enabling factors, each additional minute of consultation length was associated with an increase of $2.0 \%(95 \% \mathrm{CI}: 1.2-2,9 \%)$ in number of nurse consultations, each new medical consultation was associated with a increase of $2.7 \%$ (95\% CI: $2.1-3.2 \%$ ) and the delay in getting appointment over a day, represented a decrease of $32.8 \%(95 \% \mathrm{CI}: 19.3$ to $44.1 \%)$ in the total nursing consultations. Each chronic condition, which expresses the need health, was associated with an increase in the number of visits of $4.8 \%$ (95\% CI: 1.7 to $8.0 \%$ ). The improved perception of quality of life was associated with a reduction of $5.4 \%(95 \%$ CI 1.0 to $8.7 \%)$ of the consultations.

Conclusion: The difference of the use of primary care nurse consultations is based on health need criteria, but is also influenced by accessibility conditions.

Keyword: Health services needs and demand. Utilization. Primary health care. Nursing. Inequality. Socioeconomic factors. 


\section{INTRODUCCIÓN}

Las políticas de salud deben tener un horizonte de eficiencia pero no pueden diseñarse sin tener en consideración la accesibilidad, la solidaridad y la equidad ${ }^{1}$. La planificación de los servicios sanitarios está íntimamente ligada a sus condiciones de utilización, lo que hace preciso comprender los mecanismos que explican las diferencias en su utilización.

En al ámbito de la atención primaria (AP) se han descrito factores asociados a la utilización de la consulta del médico de familia tanto en el ámbito nacional ${ }^{2-4}$ como internacional ${ }^{5-7}$. Sin embargo, en nuestro medio, solo localmente se han estudiado los factores que influyen en la variabilidad de la utilización de la consulta de enfermería en $\mathrm{AP}^{8}$.

La primera referencia a la consulta de enfermería data de 1973, en el Johns Hopkins Hospital de Baltimore. En España este desarrollo está ligado a la reforma de la AP, que se asoció con un aumento de la utilización de los servicios prestados por la enfermería ${ }^{9}$. En nuestro entorno social, con un envejecimiento paulatino de la población y un incremento progresivo de la cronicidad, que hace necesaria la potenciación de los cuidados en la comunidad, se ha reclamado potenciar el papel de la enfermería de AP como gestora de esos cuidados ${ }^{10}$. Pero también se critica que el modelo de atención de enfermería en AP presente actualmente una distribución de los servicios no siempre relacionada con la necesidad y una atención repetida al mismo tipo de pacientes crónicos. Una oferta de servicios que no responde a las necesidades de la población y que puede ser evitada por una acción de política sanitaria constituye una inequidad en salud ${ }^{12}$.

Para evaluar si la oferta de servicios de salud se distribuye de una manera equitativa se ha recurrido a diversos análisis que relacionan el grado de accesibilidad para cada nivel de necesidad ${ }^{13,14}$. Pero una forma de estudio sistemático de la oferta de servicios requiere el marco de un modelo teórico. Existen varios tipos de modelos que conceptualizan la utilización de servicios sanitarios. Uno de los más utilizados, por su consistencia y por su carácter integrador es el "modelo conductual" propuesto por Andersen (behavioral model ${ }^{15}$. Este modelo considera unos determinantes primarios de utilización (características del entorno y de los individuos) que producen una conducta en salud (hábitos en salud y uso del sistema sanitario) que, a su vez, producen unos resultados en salud (percepción del estado de salud, un estado de salud evaluable y una satisfacción en el usuario). Los determinantes primarios, tanto del entorno como individuales, pueden agruparse en predisponentes, facilitadores y de "necesidad"16.

El modelo conductual define la utilización "equitativa" como aquella cuya variabilidad era explicada sobre todo por factores demográficos $\mathrm{y}$ de necesidad ${ }^{15}$. Desde cualquier perspectiva, la evaluación de la utilización de los servicios sanitarios no puede realizarse independientemente del nivel de necesidad, pues eso nos lleva a caracterizar conductas de una manera inadecuada, como la llamada hiperfrecuentación ${ }^{17}$.

La equidad debe constituirse en un elemento nuclear a la hora de planificar los servicios sanitarios ${ }^{1}$, incluida la consulta enfermería en AP, lo que exige el conocimiento y análisis de la situación actual de la oferta de este servicio.

El objetivo de este estudio fue conocer qué factores influyen en la utilización de la consulta de enfermería en atención primaria.

\section{SUJETOS Y MÉTODOS}

Diseño. Estudio multicéntrico transversal.

Población de estudio. En el contexto de un estudio sobre evaluación de servicios sanitarios, cuyo trabajo de campo se realizó entre octubre de 2011 y enero de 2012, se 
entrevistó a sujetos mayores de 18 años que habían acudido a consultar en 23 centros de salud de la Comunidad de Madrid ${ }^{18}$.

Tamaño muestral. El tamaño muestral se estableció en 600 sujetos aproximadamente (al menos 30 pacientes de 23 centros de salud). Por estudios previos se esperaba un coeficiente de correlación intraclase $(\rho)$ que oscilase entre 0,04 y 0,05 y se aconsejó que el número de sujetos por cluster fuera superior a $1 / \rho^{19}$. El número de cluster debía ser superior a 20 para poder construir modelos robustos con la metodología planificada (modelos mediante GEE) ${ }^{20}$.

Selección de los participantes. Los centros de salud fueron escogidos de manera que estuviesen representados los ámbitos rural (6) y urbano (17). 12 centros correspondían a zonas con rentas medias en el tercil superior y 11 se ubicaban en zonas con rentas del tercil inferior de la distribución de la Comunidad de Madrid. Dentro de cada centro la selección de participantes se realizó mediante muestreo aleatorio sistemático sobre la agenda de citación de la consulta de enfermería, 90\% sobre cita en el centro y $10 \%$ sobre cita en domicilio. Fueron excluidos aquellos pacientes que no estaban capacitados para dar su consentimiento informado.

Variables. Se consideraron factores predisponentes del entorno el hábitat (rural/urbano) y vivir en zonas de renta alta o baja. Fueron factores facilitadores el lugar de la consulta (centro de salud o domicilio), la demora para conseguir cita (en el mismo día, 1 día, 2 días, 3 días, >3 días), la demora de la consulta ( $<15$ minutos, $16-30$ minutos, 3160 minutos, $>60$ minutos) y la duración real de la consulta en minutos y el número de consultas al médico durante el año. Los factores de necesidad relativos al entorno no se midieron.

De las características personales se catalogaron como predisponentes las demográficas (edad y sexo), el número de familiares, la nacionalidad, el número de personas en el hogar y su percepción de apoyo familiar medido con el test de Apgar ${ }^{21}$. Se consideraron factores facilitadores el nivel de estudios (estudios superiores si habían terminado el bachiller o tenían estudios universitarios), la "clase social" en una clasificación de 6 categorias $^{22}$, (clase social "alta" para las dos primeras), la renta familiar en miles de euros ajustada por el número de personas del hogar (según el método propuesto por la OCDE) ${ }^{23}$, tener doble cobertura (otros seguros sanitarios diferente al público) y la consulta "en domicilio". Se consideraron factores de "necesidad" el número de condiciones crónicas (definidas como aquellas que requieren atención continuada por un periodo superior a 6 meses) y haber tenido ingresos hospitalarios en el último año.

La variable dependiente fue el número de consultas de enfermería durante el último año. Dada su asimetría y para lograr un mejor ajuste del modelo se calculó el logaritmo neperiano de este valor.

Fuentes de información. Las variables clínicas de los participantes fueron recogidas de su historia clínica informatizada . La información socioeconómica, las características de la consulta, la satisfacción y la percepción de la calidad de vida relacionada con la salud se recogieron mediante entrevista personal realizada por un profesional entrenado tras obtener el consentimiento informado escrito de cada participante. En el modelo "conductual" la satisfacción con los servicios sanitarios y la calidad de vida se consideran resultados del contacto con el sistema de salud. La satisfacción fue evaluada con un cuestionario validado con cuatro dimensiones (global, cuidados ofrecidos por el profesional, tiempo dedicado a la consulta y profundidad de la relación con el profesional ${ }^{24}$. La calidad de vida se midió con el cuestionario EuroQol-5D y sus resultados se expresaron en la escala visual y se transformaron en utilidades según la propuesta de Herdman y cols ${ }^{25}$. 
Análisis estadístico. Se realizó una descripción de los resultados mediante medidas de tendencia central y de dispersión para las variables cuantitativas y mediante los porcentajes para las variables cualitativas. Se calcularon los intervalos de confianza del 95\%.

Los sujetos se captaron desde diferentes clusters o conglomerados naturales que venían definidos por la pertenencia a determinado centro de salud. Para evaluar la asociación entre las variables personales y del entorno con la utilización de la consulta se recurrió a los modelos Generalizad Estimating Equations (GEE) los cuales corrigen la no-independencia entre los individuos de un mismo grupo y no son muy exigentes acerca de la distribución de la variable de resultado, pues ofrecen medidas de los errores de las estimaciones que son estables incluso si no se es muy preciso con la definición de las correlaciones esperadas o si la intensidad de la correlación entre las observaciones es diferente en los diferentes grupos. Su interpretación supone caracterizar el promedio de respuesta poblacional cuando cambian las variables independientes en el conjunto de la población ${ }^{26}$.

Se construyó un modelo para factores predisponentes, otro para los facilitadores, un tercero para factores de necesidad y un modelo final que incluyó las variables de calidad de vida y satisfacción. Los modelos presentados se seleccionaron entre todos los posibles por su coherencia con el modelo teórico y por el principio de parsimonia, es decir, entre dos posibles modelos similares se elige el que sea más sencillo y que menos suposiciones necesite para su construcción.

\section{RESULTADOS}

El número de participantes fue de 662 , cuyas características se recogen en la tabla 1. Rechazaron participar 95, los cuales no presentaron diferencias en su edad media ni en el porcentaje de varones/mujeres que los que aceptaron.

En la tabla 2 se incluyen las características de la valoración de la consulta y la satisfacción de los participantes.

\section{Tabla 1}

\section{Características de los sujetos incluidos}

\begin{tabular}{|c|c|c|c|}
\hline & Media (DS) & Mediana (rango IC) & Porcentajes (IC 95\%) \\
\hline Edad en años & $65,4(16,6)$ & $69(55-78)$ & \\
\hline Sexo (mujer) & & & $60,7 \%(56,9-64,5 \%)$ \\
\hline Nacionalidad española & & & $95,2 \%(93,5-96,9 \%)$ \\
\hline Condiciones crónicas $\left(\mathrm{n}^{\circ}\right)$ & $2,7(2,3)$ & $2(1-4)$ & \\
\hline Condiciones crónicas (sí) & & & $82,9 \%(79,9-85,9 \%)$ \\
\hline EVA - EuroQol-5-D & $65,6(22,3)$ & $70(50-80)$ & \\
\hline EuroQol-5-D Utilidades & $0,68(0,28)$ & $0,76(0,48-1,00)$ & \\
\hline Otro aseguramiento & & & $16,3 \%(13,4-19,2 \%)$ \\
\hline $\mathrm{N}^{\mathrm{o}}$ de familiares en el hogar & $2,6(1,4)$ & $2(2-3)$ & \\
\hline Grupo social alto & & & $22,4 \%(19,2-25,5 \%)$ \\
\hline Estudios superiores & & & $37,2 \%(33,5-, 40,9 \%)$ \\
\hline Renta familiar ajustada (mil€) & $\begin{array}{c}0,873 \\
(0,514) \\
\end{array}$ & $\begin{array}{c}0,707 \\
(0,600-1,000) \\
\end{array}$ & \\
\hline
\end{tabular}

DS: Desviación estándar; IC 95\%: Intervalo de confianza del 95\%; Rango IC: rango intercuartílico (percentil 25percentil 75). EVA-EuroQol-5_D. Escala visual analógica del cuestionario EuroQol-5D 
Tabla 2

Características de la consulta de enfermería tras la que se incluyó a los participantes

\begin{tabular}{|c|c|c|c|}
\hline & Porcentajes (IC 95\%) & Media (DS) & Mediana (rango IC) \\
\hline Origen de la demanda & $24,3 \%(21,0-27,7 \%)$ & & \\
\hline Autoconcertado & $65,4 \%(61,7-69,1 \%)$ & & \\
\hline Derivado por otra enfermera & $0,5 \%(0,1-1,3 \%)$ & & \\
\hline Derivado por otros & $9,8 \%(7,5-12,2 \%)$ & & \\
\hline \multicolumn{4}{|l|}{ Tiempo en conseguir consulta } \\
\hline En el mismo día & $81,3 \%(78,2-84,3 \%)$ & & \\
\hline Un día & $6,3 \%(4,4-8,3 \%)$ & & \\
\hline Dos días & $4,8 \%(3,1-6,5 \%)$ & & \\
\hline Tres días & $1,8 \%(0,7-2,9 \%)$ & & \\
\hline Más de tres días & $5,7 \%(3,9-7,6 \%)$ & & \\
\hline \multicolumn{4}{|l|}{ Tiempo de espera en consulta } \\
\hline Menos de 15 minutos & $84,9 \%(82,1-87,7 \%)$ & & \\
\hline Entre 16 y 30 minutos & $12,1 \%(9,5-14,6 \%)$ & & \\
\hline Entre 31 y 60 minutos & $2,65(1,3-3,8 \%)$ & & \\
\hline Más de una hora & $0,5 \%(0,1-1,3 \%)$ & & \\
\hline Duración de la consulta (min) & & $13,3(7,4)$ & $10(10-15)$ \\
\hline Satisfacción global* & & $4,89(0,42)$ & $5,00(5,00-5,00)$ \\
\hline Satisfacción con cuidados* & & $4,76(0,49)$ & $5,00(4,75-5,00)$ \\
\hline Satisfacción con el tiempo dedicado* & & $4,48(0,70)$ & $5,00(4,00-5,00)$ \\
\hline Satisfacción con la relación con el profesional $*$ & & $3,34(1,45)$ & $3,67(2,33-4,67)$ \\
\hline
\end{tabular}

IC 95\%: Intervalo de confianza del 95\%; DS: Desviación estándar; Rango IC: rango intercuartílico (percentil 25-percentil 75). * (1 la peor puntuación, 5 la mejor posible )

El número medio de consultas de enfermería en el último año fue de 17,6 (IC95\%: 15,6-19,7) con una mediana de 11 y un rango intercuartil de 6-17. Para las consultas médicas el número medio en el último año fue de 11,6 (IC 95\%: 10,8$12,5)$, con una mediana de 9 y rango intercuartil de 4-15).

En la tabla 3 se recogen las características explicativas agrupadas por su papel predisponente, facilitador y de necesidad. Dentro del modelo de los factores facilitadores, la renta familiar ajustada se asoció negativamente con el número de consultas de enfermería, disminuyendo una media del 13,4\% su número (IC95\%: 1,9-23,7\%) por cada mil euros que aumentaba la renta familiar (datos no presentados). En la tabla 4 se recogen los resultados del modelo final. La renta de la zona (factor predisponente del entorno) que no había sido mantenida en el modelo de la tabla 3, mejoró el ajuste del modelo final por lo que fue incluida en este. Vivir en un barrio de renta alta aumentó la media del número de consultas de enfermería en un $17 \%$ (IC 95\%: $0,4-36,9 \%$ ).

Entre los factores predisponentes, cada aumento de la edad en diez años se asoció con un aumento en la media del número de consultas de enfermería del 11,0\% (IC95\%:6,2-16,2\%).

De los factores facilitadores, se asociaron con un aumento del número medio de consultas el tiempo empleado en la ultima consulta (aumentando un 2,0\% por cada minuto dedicado; IC95\%:1,2-2,9\%) y el 
Tabla 3

Modelos explicativos de la variabilidad del uso de la consulta para los factores predisponentes, facilitadores y de "necesidad"

\begin{tabular}{|c|c|c|c|c|}
\hline \multicolumn{5}{|c|}{ Factores predisponentes } \\
\hline Variable & Coeficiente & $\mathrm{p}$ & LIIC 95\% & LSIC 95\% \\
\hline Edad & 0,020 & $<0,001$ & 0,0154764 & 0,024 \\
\hline Español & 0,278 & 0,096 & $-0,0495722$ & 0,606 \\
\hline Puntuación Apgar & $-0,027$ & 0,102 & $-0,0598116$ & 0,005 \\
\hline $\mathrm{N}^{\mathrm{o}}$ familiares en el hogar & 0,065 & 0,020 & 0,0100916 & 0,120 \\
\hline Constante & $0, .851$ & 0,001 & 0,3468342 & 1,354 \\
\hline \multicolumn{5}{|l|}{$\begin{array}{l}\mathrm{N}=661 \\
\text { Wald chi } 2(4)=96,19 . \text { Prob }<0, .0001\end{array}$} \\
\hline \multicolumn{5}{|c|}{ Factores facilitadores } \\
\hline Variable & Coeficiente & $\mathrm{p}$ & LIIC 95\% & LSIC 95\% \\
\hline Consulta en domicilio & 0,473 & $<0,001$ & 0,251 & 0,695 \\
\hline Retraso en la cita superior a 24 horas & $-0,421$ & $<0,001$ & $-0,613$ & $-0,228$ \\
\hline Duración consulta enfermería & 0,019 & $<0,001$ & 0,009 & 0,029 \\
\hline $\mathrm{N}^{\circ}$ consultas médico familia & 0,029 & $<0,001$ & 0,023 & 0,034 \\
\hline Estudios superiores & $-0,248$ & $<0,001$ & $-0,381$ & $-0,115$ \\
\hline Constante & 1,377 & $<0,001$ & 1,126 & 1,627 \\
\hline \multicolumn{5}{|l|}{$\begin{array}{l}\mathrm{N}=661 \\
\text { Wald chi2 }(5)=253,98 . \text { Prob }<0,0001\end{array}$} \\
\hline \multicolumn{5}{|c|}{ Factores de "necesidad" } \\
\hline Variable & Coeficiente & $\mathrm{p}$ & LIIC 95\% & LSIC 95\% \\
\hline Hospitalizados en el año & & $<0,001$ & 0,133 & 0,431 \\
\hline $\mathrm{N}^{\mathrm{o}}$ de condiciones crónicas & & $<0,001$ & 0,108 & 0,169 \\
\hline Constante & & $<0,001$ & 1,743 & 2,014 \\
\hline $\begin{array}{l}N=647 \\
\text { Wald } \operatorname{chi} 2(3)=107,14 . \text { Prob }<0,0001\end{array}$ & & & & \\
\hline
\end{tabular}

Variable dependiente $\ln$ ( $\mathrm{n}^{\circ}$ consultas de enfermería/año). LIIC 95\%: Límite inferior del Intervalo de confianza del $95 \%$

número de consultas anuales al médico de familia (cada nueva consulta médica se asoció con un aumento medio del $2,7 \%$ de las consultas de enfermería (IC95\%: 2,1-3,2\%). Una demora de más de 24 horas en conseguir cita se asoció con una disminución del 32,8\% del número medio de consultas (IC95\%: 19,3$44,1 \%$ ). Tener otro seguro médico se relacionó con una disminución media del $12,9 \%$ de las consultas, aunque esta asociación no fue estadísticamente significativa $(\mathrm{p}=0,107)$.

Entre los elementos relacionados con la necesidad, cada nueva condición crónica se asoció con un incremento medio del número de consultas del 4,8\% (IC 95\%: 1,7$8,0 \%)$.

Cada cambio de quintil en la percepción de la calidad de vida relacionada con la salud se asoció con una reducción media del número de consultas de un 5,4\% (IC95\%: $1,0-8,7 \%$ ). Pasar del grado más bajo de satisfacción en la relación con el profesional al más alto se asoció con un aumento medio del número de consultas del 17,3\% (IC 95\%:9,9-39-4\%). 
Tabla 4

Modelo explicativo final de las diferencias en la utilización de la consulta de enfermería

\begin{tabular}{|l|c|c|c|c|}
\hline Variable & Coeficiente & $\mathrm{p}$ & LIIC 95\% & LSIC 95\% \\
\hline Barrio de renta alta & 0,159 & 0,045 & 0,004 & 0,314 \\
\hline Edad & 0,010 & 0,006 & 0,006 & 0,015 \\
\hline Otro aseguramiento & $-0,138$ & 0,107 & $-0,305$ & 0,030 \\
\hline Retraso en la cita superior a 24 horas & $-0,398$ & $<0,001$ & $-0,580$ & $-0,215$ \\
\hline Duración consulta enfermería & 0,020 & $<0,001$ & 0,012 & 0,029 \\
\hline $\mathrm{N}^{\text {o consultas médico familia }}$ & 0,027 & $<0,001$ & 0,021 & 0,032 \\
\hline $\mathrm{N}^{\text {o de condiciones crónicas }}$ & 0,047 & 0,002 & 0,017 & 0,077 \\
\hline Satisfacción con la relación con enfermería* & 0,040 & 0,067 & $-0,003$ & 0,083 \\
\hline Utilidad en quintiles** & $-0,055$ & 0,018 & $-0,101$ & $-0,009$ \\
\hline Constante & 0,984 & 0,000 & 0,602 & 1,367 \\
\hline $\begin{array}{l}\mathrm{N}=646 \\
\text { Wald chi2(4)= 344,11. Prob }<0,0001\end{array}$ & & & \\
\hline
\end{tabular}

Variable dependiente $\ln \left(\mathrm{n}^{\circ}\right.$ consultas de enfermería/año). LIIC 95\%: Límite inferior del Intervalo de confianza del 95\%. LSIC 95\%: Límite superior del Intervalo de confianza del 95\%. * 1 la peor relación posible- 5 la mejor relación posible. ** Calidad de vida relacionada con la Salud expresada en el EuroQol-5D y transformada en utilidades. Se divide la distribución en quintiles.

\section{DISCUSIÓN}

La utilización de la consulta de enfermería es muy variable en nuestro entorno y las diferencias pueden explicarse, en parte, por las características de los pacientes y las del medio en el que se desarrolla el proceso de atención, lo que puede tener implicaciones al analizar la equidad en la oferta del servicio.

Al valorar los factores predisponentes independientemente, encontramos que el apoyo familiar se asocia con un menor número de consultas, como se recoge en la literatura ${ }^{2}$, aunque las familias con más miembros realizan una mayor utilización, quizá debido a la estructura familiar de nuestro medio, donde una persona, fundamentalmente la madre, hace consultas para varios miembros de la familia. Ser inmigrante se asoció con un menor número de consultas, lo que se había descrito previamente para la consulta de enfermería ${ }^{27}$, no siendo tan clara esta relación para el conjunto del sistema sanitario ${ }^{28}$. Además esta asociación no se mantiene en el modelo final. La edad es el factor predisponente que más consistentemente se asocia con el uso de la consulta de enfermería, circunstancia recogida en previamente en la literatura para la consulta médica $^{3,4,14}$.

Del análisis de los factores facilitadores por separado se observa que todos aquellos que implican mayor accesibilidad se asocian de manera directa con una mayor utilización y que un mayor nivel de estudios (o una mayor renta familiar) se asocian con un menor uso de este servicio, lo que ya se había observado en nuestro medio para la consulta del médico de familia ${ }^{3,4}$.

Pero estas asociaciones deben valorarse en el contexto del modelo final. En él encontramos que se asocia con la utilización una variable predisponente del entorno (la renta de la zona), que la edad permanece como elemento explicativo importante y que mantienen su peso los llamados factores facilitadores relacionados con la accesibilidad y los factores de necesidad. Poseer otro aseguramiento explica una ten- 
dencia a utilizar menos el servicio. Además la calidad de vida relacionada con la salud (CVRS) aparece como otro elemento explicativo fundamental y una dimensión de la satisfacción, la que tiene que ver con la relación con el profesional también podría explicar las diferencias en la utilización. En el modelo conductual estas dos últimas variables se consideran de "resultado", aunque se acepta que modifican a su vez la utilización de servi$\operatorname{cios}^{16}$. Desde una perspectiva amplia podríamos considerar que la satisfacción actúa como facilitador de la utilización y la CVRS como un "proxy" de la necesidad percibida .

Si intentamos valorar estos resultados desde el punto de vista de la equidad, el peso explicativo que tienen los factores relacionados con la necesidad (incluida la CVRS) hablan a favor de una distribución equitativa y en contra de ello están los factores que tienen que ver con la accesibilidad.

La asociación entre los factores de necesidad y las variaciones en el uso de servicios es consistente y acorde con lo esperado desde el punto de vista de una planificación sanitaria ${ }^{1}$. Sin embargo, las diferencias en el uso asociadas a factores de accesibilidad generan desigualdades en salud y constituyen un elemento fundamental en la inequidad en salud ${ }^{12}$. Aunque menos del 13\% de los participantes tardan más de un día en conseguir citas, es este grupo el que hace una menor utilización del servicio. Tampoco parecen inadecuados los tiempos medios de duración de la consulta (alrededor de 13 minutos), pero una vez ajustado por necesidad siguen utilizando más las consultas aquellos a quien más tiempo se dedica en cada una, lo que nos lleva a esa situación indeseada de falta de eficiencia del servicio ${ }^{11}$. La asociación entre el número de consultas médicas y de enfermería puede ser más compleja de interpretar en el marco teórico utilizado. Un mayor número de consultas médicas supone una mayor accesibilidad al sistema pero también puede expresar también un mayor nivel de necesidad. La influencia de la accesibilidad en la variabilidad de la utilización de la con- sulta de enfermería es novedosa respecto a lo observado con otros servicios en AP en nuestro medio ${ }^{4}$.

El mayor uso de los servicios de enfermería en barrios de renta alta puede ser resultado de una menor frecuentación en estas zonas ${ }^{3,4}$, que a su vez facilite una mayor accesibilidad, más que deberse a una inequidad por renta. A favor de esta idea está el hecho de que la renta familiar o un mayor nivel de estudios se asocien con menos visitas en el modelo de factores facilitadores. Lo que no se ha observado en el uso de la consulta de enfermería es esa inequidad pro-pobres (discriminación positiva), descrita para la utilización de otro tipo de consultas en AP en los sistemas públicos de salud 3 ,4,6, 30-33. Tampoco se han observado desigualdades en el uso de servicios relativas al sexo. Se había descrito un mayor uso de consultas de médicos de AP en el sistema público por muje$\mathrm{res}^{34}$, circunstancia que no se da en nuestro estudio.

Las limitaciones del trabajo se relacionan con el diseño elegido. Dado que la selección de los participantes se realizó sobre los listados de citaciones, estarán sobrerrepresentados los que hacen una utilización más intensiva. Además la naturaleza de las asociaciones no puede ser interpretada en términos de causa-efecto por tratarse de un estudio transversal. El marco teórico elegido permite la interpretación de modelos explicativo o $\operatorname{predictivos}^{15}$, pero no se pueden efectuar predicciones ni establecer relaciones de causalidad.

Por otra parte, algunos factores relacionados con la accesibilidad, como la duración de la consulta, o el tiempo en conseguir cita, se refieren a la última consulta, que solo de manera probabilística se relacionan con el tiempo medio de consulta o con las dificultades para ser citado

Entre las fortalezas del estudio se deben señalar las relacionadas con la calidad de la información. La utilización de registros 
informatizados para conocer el número de visitas, las patologías y los ingresos hospitalarios evita la presencia de sesgos de memoria y la recogida de datos individuales por una misma entrevistadora entrenada al efecto, ofrece una calidad sobre los datos sociodemográficos del mayor nivel posible.

La aplicabilidad de estos resultados en el ámbito de la AP es inmediata. La consolidación del necesario papel de la enfermería de AP como gestora de los cuidados, especialmente en las patologías crónicas, exige cuidar especialmente los problemas de accesibilidad al servicio. Corresponde a quien los planifica junto a aquellos que lo prestan, mejorar estas circunstancias.

En conclusión, el modelo conductual nos ofrece un marco de referencia adecuado para estudiar tanto la utilización de los servicios sanitarios como la equidad de este uso. Parece que la variabilidad del uso de servicios de enfermería en AP responde fundamentalmente a criterios de necesidad, pero factores relacionados con la accesibilidad tienen un peso importante en las diferencias de utilización. El peso de los factores socioeconómicos en las diferencias de utilización del servicio es muy escaso, pero no podemos hablar de una utilización plenamente equitativa mientras no se eliminen las diferencias en el acceso al servicio.

\section{BIBLIOGRAFÍA}

1. Reidpath DD, Olafsdottir AE, Pokhre S, Allotey P The fallacy of the equity-efficiency trade off: rethinking the efficient health system. BMC Public Health. 2012; 12(Suppl 1):S3.

2. Bellón JA, Delgado-Sánchez A, de Dios Luna J, Lardelli-Claret P. Patient psychosocial factors and primary care consultation: a cohort study. Fam Pract. 2007; 24: 562-569.

3. Pérez MA, Moreno VM, Puerta DR, Martínez YG, Vicario IH, Ceruelo EE, et al. Factores socioeconómicos y frecuentación en las consultas de medicina de familia de la red sanitaria pública madrileña. Gac Sanit. 2007;21: 219-226.
4. Martín-Fernández J, Gómez-Gascón T, Cura-González MI, Tomás-García N, Vargas-Machuca C, Rodríguez-Martínez G. La calidad de vida relacionada con la salud como factor explicativo de la utilización de la consulta de medicina de familia: un estudio bajo el modelo conductual. Rev Esp Salud Publica. 2010 84: 309-319.

5. Carr-Hill RA, Rice N, Roland M.. Socioeconomic determinants of rates of consultation in general practice based on fourth national morbidity survey of general practices. BMJ. 1996; 312: 1008-1012.

6. Health Utilisation Research Alliance (HURA). Ethnicity, socioeconomic deprivation and consultation rates in New Zealand general practice. J Health Serv Res Policy. 2006;11:141-149.

7. Smits FT, Brouwer HJ, ter Riet G, van Weert HC. Epidemiology of frequent attenders: a 3-year historic cohort study comparing attendance, morbidity and prescriptions of one-year and persistent frequent attenders. BMC Public Health. 2009; 9:36.

8. Gálvez-Romero C, González-Valentín A, RamónGarrido ED. Estado de salud y utilización de recursos sanitarios de una población de ancianos atendidos en domicilio. Gerokomos. 2007; 18: 15-24.

9. Fernández JL, Bolíbar I; López J, Sanz E. El impacto de la reforma de la atención primaria en la utilización de servicios médicos y de enfermería. Aten Primaria. 1996;18:53-57.

10. Contel JC, Muntané B, Camp L. La atención al paciente crónico en situación de complejidad: el reto de construir un escenario de atención integrada. Aten Primaria. 2012; 44:107-113.

11. Martínez-Riera JR. Consulta de enfermería a demanda en Atención Primaria. Reflexión de una necesidad. Rev Adm Sanit. 2003; 1:425-440.

12. Braveman P. Health disparities and health equity: Concepts and measurement. Annu Rev Public Health. 2006; 27:167-194

13. Mendoza-Sassi R, Béria JU. Utilización de los servicios de salud: una revisión sistemática sobre los factores relacionados. Cad Saúde Pública. 2001; 17: 819-832.

14. Van Doorslaer E, Masseria C, Koolman X: Inequalities in access to medical care by income in developed countries. CMAJ. 2006; 174:177-183.

15. Andersen RM. Revisiting the behavioral model and access to medical care: does it matter? J Health Soc Behav. 1995; 36:1-10. 
16. Andersen RM. National Health Surveys and the Behavioral Model of Health Services Use. Med Care. 2008; 46: 647-653.

17. Luciano Devis JV, Serrano Blanco A, Grupo DASMAP. Los hiperfrecuentadores en atención primaria: perfil sociodemográfico, características clínicas y propuesta de una nueva definición. Aten Primaria. 2008; 40: 631-632.

18. Martín-Fernández J , del Cura-González MI, Rodríguez-Martínez G, Ariza-Cardiel G, Zamora J, et al. Economic Valuation of Health Care Services in Public Health Systems: A Study about Willingness to Pay (WTP) for Nursing Consultations. PLoS ONE. 2013; 8(4): e62840.

19. Donner A, Klar N. Pitfalls of and controversies in cluster randomized trials. Am J Public Health. 2004; 94:416-422.

20. Feng Z, McLaran D, Grizzle J. A comparison of statistical methods for clustered data analysis with Gaussian error. Stat Med. 1996; 15:1793-1806.

21. Bellón Saameño JA, Delgado Sánchez A, Luna del Castillo JD, Lardelli Claret P. Validez y fiabilidad del cuestionario de función familiar Apgarfamiliar. Aten Primaria. 1996;18: 289-296.

22. Domingo Salvany A, Marcos Alonso J. Propuesta de un indicador de la" clase social" basado en la ocupación. Gac Sanit. 1989; 3: 320-326.

23. Hagenaars, A. de Vos K. and Zaidi MA. Poverty Statistics in the Late 1980s: Research Based on Microdata. Luxembourg: Office for Official Publications of the European Communities; 1994.

24. Fernández San Martín MI, Rebagliato Nadal O, de Gispert Uriach B, Roig Carrera H, Artigas Guix J, Bonay Valls B, et al. Adaptación de un cuestionario de satisfacción del paciente con la consulta médica y de enfermería. Aten Primaria. 2008; 40:611-616.

25. Herdman M, Badia X, Berra S. El EuroQol5D: una alternativa sencilla para la medición de la calidad de vida relacionada con la salud en atención primaria. Aten Primaria. 2001; 28: 425-30.

26. Hanley JA, Negassa A, Edwardes MD, Forrester JE. Statistical analysis of correlated data using generalized estimating equations: an orientation. Am J Epidemiol. 2003;157: 364-375.
27. Soler-González J, Serna Arnáiz C, Rué Monné M, Bosch Gaya A, Ruiz Magaz MC, Gervilla Caño J. Utilización de recursos de atención primaria por parte de inmigrantes y autóctonos que han contactado con los servicios asistenciales de la ciudad de Lleida. Aten Primaria. 2008; 40: 225231 .

28. Regidor E, Sanz B, Pascual C, Lostao L, Sánchez E, Díaz Olalla JM.. La utilización de los servicios sanitarios por la población inmigrante en España. Gac Sanit. 2008; 23: 4-11.

29. Sáez M. Condicionantes en la utilización de los servicios de atención primaria: Evidencias empíricas e inconsistencias metodológicas. Gac Sanit. 2003; 17: 412-419.

30. Lostao L, Regidor E, Calle M E, Navarro P, Domínguez V. Evolución de las diferencias socioeconómicas en la utilización y accesibilidad de los servicios sanitarios en España entre 1987 y 1995/97. Rev Esp Salud Publica. 2001; 75: 115-128.

31. Vedsted P, Olesen F. Social environment and frequent attendance in Danish general practice. Br J Gen Pract. 2005; 55:510-515.

32. Regidor E, Martínez D, Calle ME, Astasio P, Ortega P, Domínguez V. Socioeconomic patterns in the use of public and private health services and equity in health care. BMC Health Serv Res. 2008;8:183.

33.Crespo-Cebada E, Urbanos-Garrido RM. Equity and equality in the use of GP services for elderly people: The Spanish case. Health Policy 2012; 104: 193199.

34. Rodríguez M, Stoyanova A. La influencia del tipo de seguro y la educación en los patrones de utilización de los servicios sanitarios. Gac Sanit. 2004;18(Supl 1):102-111 